\title{
Les protections patrimoniales au titre des sites et paysages
}

Catherine Bergeal

\section{(2) OpenEdition \\ Journals}

Édition électronique

URL : https://journals.openedition.org/rhcf/576

DOI : $10.4000 /$ rhcf. 576

\section{Éditeur}

Rails \& histoire

Édition imprimée

Date de publication : 1 novembre 2005

Pagination : 113-118

ISSN : 0996-9403

Référence électronique

Catherine Bergeal, «Les protections patrimoniales au titre des sites et paysages », Revue d'histoire des chemins de fer [En ligne], 32-33 | 2005, mis en ligne le 30 mars 2011, consulté le 22 avril 2022. URL http://journals.openedition.org/rhcf/576 ; DOI : https://doi.org/10.4000/rhcf.576 


\section{Catherine BERGEAL}

\section{Les protections patrimoniales au titre des sites et des paysages}

Je remercie l'Association pour l'histoire des chemins de fer en France de me donner l'occasion de re-visiter pratiquement la totalité du champ de ma sous-direction. En effet, la thématique abordée aujourd'hui est l'une des plus riches et des plus complexes et elle illustre bien l'ensemble des activités que la sous-direction des Sites et Paysages a à traiter. Ainsi, je ne vous parlerai pas uniquement des protections des sites établies par la loi de 1930 - en fait déjà par la loi de 1906 - qui sont le pendant des protections instituées par la loi de1913 pour les monuments historiques. Je préfère vous restituer ce que j'ai ressenti à l'écoute des débats qui se sont succédé jusqu’à présent, par lesquels vous avez parfaitement exprimé ce que sont nos politiques en matière de sites et de paysages. Pour moi, les deux domaines sont totalement imbriqués. Le patrimoine et les politiques qualitatives en matière de paysage concernent l'ensemble de nos territoires, qu'ils soient naturels ou urbains. Nous considérons la totalité de nos paysages et non seulement la nature.

Je vais vous faire part d'une conviction : le paysage c'est, d'abord, du temps, de l'épaisseur historique traduits dans l'espace. Quand on prend du recul, on voit combien les paysages ont été «malaxés » par l'homme et par nos activités humaines. Or, la constitution du réseau du chemin de fer, en seulement trente à cinquante ans, a représenté sans doute le phénomène le plus marquant de l'évolution du territoire et des paysages français. On voit combien la France a été marquée par lui, jusque dans ses derniers petits villages.

Nous avons connu peu de phénomènes aussi rapides, aussi prégnants et qui se sont révélés aussi forts dans la structuration de nos peuplements et de notre organisation territoriale, par rapport :

- à la possibilité de développement de l'industrie,

- aux modes de déplacements,

- et, on l'a vu, à la perception et à l'appropriation par nos concitoyens de ce que sont nos territoires et la diversité des paysages français. 
Avec l'essor du tourisme, l'histoire des chemins de fer a conduit à un bouleversement complet de l'occupation de nos territoires, de leur perception et de leur appropriation collective. En outre, cette histoire nous permet d'apprécier de près les conséquences de certaines politiques publiques. À titre d'exemple, l'actualité qui nous concerne aujourd'hui est celle des politiques de l'énergie éolienne, en termes de rapidité du phénomène et d'impact sur nos paysages. Toutefois, il faut garder le sens des proportions dans cette analogie car les éoliennes ne vont pas modifier nos déplacements, générer de nouveaux lieux de créations d'entreprises, inverser l'évolution des villes par rapport à une nouvelle gare, ni modifier les structures foncières. Il me semble extrêmement important de le préciser et de considérer la dimension de la gestion durable de nos territoires.

Tout cela m'amène à évoquer les paysages du quotidien dont je vous parlais avec, au milieu d'eux, des éléments de valeur exceptionnelle, comme le conçoit la loi de 1930. Il s'agit de s'intéresser à l'image de marque de tous nos territoires, de regarder comment les paysages évoluent, qui sont les responsables de leur gestion, et traiter de leurs qualités avec tous les acteurs. C'est là, pour moi, le fil directeur de toutes nos politiques. Dans ce cadre, les impacts sur les paysages des politiques publiques sur l'agriculture sont essentiels $-80 \%$ de nos territoires sont ruraux. Or les évolutions de l'agriculture entrainent aujourd'hui une simplification, voire une banalisation qu'on souhaite infléchir pour préserver la diversité de nos paysages.

Les politiques publiques que nous menons en matière de qualité des paysages doivent être adaptées aux différentes échelles à traiter. Elles ont aussi bien pour objectif :

- d'encourager des communes à qualifier leurs paysages, à se les approprier, notamment pour établir leurs documents d'urbanisme ;

- de permettre un contrôle accru de la qualité des constructions par l'institution des volets paysagers, politique que l'on a tenté d'évaluer pour les permis de construire ;

- de promouvoir le recours à des professionnels compétents, notamment pour la prise en compte du paysage dans les études d'impact. À titre d'exemple, pour réaliser des projets éoliens, nous défendons le principe qu'il convient de recourir à des paysagistes et non seulement à des ingénieurs, même si leurs compétences sont parfaites. Quand un projet « fait paysage » de façon aussi forte, il est très important de faire appel à des professionnels du paysage. 
Un deuxième élément, et non des moindres, de nos politiques consiste dans le débat démocratique qui est nécessaire pour décider de l'évolution des paysages. Un élu, un citoyen, vous, tout le monde possède la légitimité nécessaire au débat. Ce n'est pas aux spécialistes, aux paysagistes de décider, seuls, de nos sorts collectifs, de nos desseins et dessins collectifs. Le débat démocratique est un fait essentiel qui croît en Europe. Il ne concerne pas uniquement la France : la Convention européenne du paysage qui devrait être ratifiée par le Parlement mentionne bien des paysages perçus par les populations, d'où la nécessité d'un débat légitime, normal, pour s'accorder sur ces valeurs à partager.

Dans ce contexte, la loi de 1930 permet de reconnaitre une valeur « exceptionnelle », c'est-à-dire reconnue par le plus grand nombre, le maintien des valeurs reconnues localement étant tout aussi indispensable à la qualité des paysages français. Or, on l'a vu, à travers notre regard sur les paysages ferroviaires, on parle bien de valeur partagée par un grand nombre de personnes, notamment des voyageurs, qui, effectivement, génère une appropriation de valeurs collectives qui vont parfois faire débat et susciter des demandes de protection ou de conservation.

Pour moi, on rejoint là un des sous-ensembles des politiques de paysage où, depuis 1906 - ce qui permet de les apprécier avec bientôt une centaine d'années de recul - on a eu l'ambition, en France, de préserver des "paysages patrimoniaux », à savoir les sites " classés ou inscrits », des secteurs sauvegardés... Or, ces politiques de conservation ne se résument pas à une simple «mise sous cloche » de paysages. Ces paysages, qui ne sont jamais naturels, ont une histoire et des caractéristiques originales dont on va débattre pour essayer de leur reconnaitre une valeur à préserver pour les générations futures. Chaque site ainsi est unique et il existe des disparités très importantes entre eux. Lorsqu'il s'agit de la montagne Sainte-Victoire dont, si vous en connaissez l'histoire, vous savez que des villages entiers ont disparu au fil des siècles, le classement a d'abord reconnu la force de cette montagne sublimée par Cézanne, force que l'homme aura beaucoup de mal à détruire. En effet, certains sites résisteront sans peine, alors que d'autres sont véritablement liés aux activités humaines qui les façonnent. C'est le cas des marais de Guérande qui sont aujourd'hui préservés dans leur totalité. Dans ce cas, l'intérêt du classement est largement débattu pour estimer la valeur et le sens de cette protection et des conditions de sa gestion dans le temps. 
Ces politiques patrimoniales sont donc exceptionnelles. Elles concernent, au niveau des sites classés, à peu près $1,4 \%$ du territoire national. Les classements seront poursuivis, mais sans aller beaucoup plus loin, l'objectif n'étant pas de couvrir $20 \%$ du territoire de la France, comme l'ambitionnent les parcs naturels régionaux. On souhaite seulement mettre en « référence » des éléments représentatifs de ce que la France offre ou veut donner comme image de la diversité de ses paysages et de leurs valeurs patrimoniales.

Cette démarche est identique à celles qui sont menées pour les monuments historiques avec lesquels nos politiques sont étroitement coordonnées. En effet, je travaille quotidiennement avec les services du ministère chargé de la Culture puisque nos outils se complètent. Dès lors qu'une valeur patrimoniale a été identifiée pour des raisons d'ordre historique, esthétique, scientifique selon les critères déterminés par les lois, on utilisera l'outil de protection le plus adapté selon qu'il s'agit d'espaces bâtis ou non. Ainsi, un ensemble urbain constitué ne sera pas a priori classé au titre de la loi de 1930, alors que certains lotissements bâtis pourront bénéficier de cette protection, d'où l'importance d'un travail technique dont je vous épargne les détails.

Ce qui est important, c'est de connaitre la valeur de ce que l'on envisage de classer. Un site est généralement un nom, c'est d'abord une identité, c'est un lieu. Pour certains, il est facile de les déterminer : une vallée, un endroit dessiné par la géographie ; pour d'autres, un travail technique complexe est nécessaire pour ce faire mais un site est toujours, en premier, un nom. Ce ne peut être ni la parcelle A7-20 ni un hectare. Nous ne sommes pas dans appréciation quantitative, mais entièrement qualitative, en sachant toutefois que les notions de sites ont peu évolué depuis cent ans, contrairement à ce que j’en pensais quand je suis arrivée dans cette sous-direction.

Toutefois, les protections au titre de la loi de 1930 ont aussi leur histoire. Par exemple, lorsqu'on préservait les gorges du Tarn lors du développement du tourisme, on préservait les points sublimes, donc les belvédères, les lieux où il se passait quelque chose, là où l'économie touristique - à l'époque les marchands de souvenirs et de cartes postales se développait, là où l'on venait consommer du paysage. Or, à présent, nous classons l'ensemble des gorges, nous classons les paysages perçus. Cela a été aussi le cas pour le site de Monségur pour lequel on a classé l'ensemble des paysages perçus du promontoire, seul classé initialement. Aujourd'hui, les protections établies cherchent à cerner les sites dans leur globalité selon des valeurs précisées dans les dossiers de protection. 
Aussi, au cours de ces vingt dernières années, en terme de surface, avons-nous préservé $60 \%$ des $1,4 \%$ du territoire national que je viens d'évoquer.

Si l'on revient à notre sujet, j’ai la conviction que certains paysages ferroviaires, comme tout autre paysage, comme tout autre objet, vont devoir être préservés comme étant un de ces joyaux exceptionnels de notre patrimoine commun. Bien entendu, pour une grande part, quand il s'agit par exemple d'un viaduc isolé remarquable par l'exploit technique qu'il représente, ils seront protégés plutôt au titre de la loi de 1913 ; en revanche quand il s'agira non seulement d'un ouvrage, peut être moins intéressant ou qui n'a pas la même valeur de référence d'un point de vue technique, mais qu'il sera particulièrement en adhésion avec son site, ce sera le « site du viaduc » qui deviendra objet de paysage et c'est la loi de 1930 qui pourra être appliquée.

Je précise que cette protection ne gérant pas «d'abords » comme celle de 1913, l'outil correspondant ayant disparu de notre arsenal réglementaire - il s'agissait des zones « titre III »-j’accepte de considérer que la loi de 1930 puisse être également utilisée pour protéger des abords de certains sites classés. Toutefois, on avance en douceur car cette notion est délicate à manier. Peut-on simplement protéger l'écrin d'un objet sans considérer que c'est l'écrin qui devient le joyau ? Les deux approches sont souvent possibles et il s'agit à mon avis chaque fois d'un cas d'espèce à analyser.

Enfin, en matière de protection d'itinéraires, une question similaire s'est déjà posée. Il s'agit du problème de la protection des chemins de Saint-Jacques de Compostelle qui relèvent de protections patrimoniales qui dépassent le niveau national, puisqu'ils sont reconnus comme du patrimoine mondial « exceptionnel et universel ». En effet, les chemins de Saint-Jacques doivent bénéficier d'un renforcement de protection selon l'évaluation de leur gestion actuellement engagée avec d'autres pays européens. Or, dans ce cadre, j'accepte complètement le principe d'utiliser la loi de 1930 pour préserver des «itinéraires ». J'ai bien noté ce matin, dans l'intervention de nos collègues suisses, qu'il faudra rechercher avec eux le fil directeur qu'on pourra adopter pour mieux valoriser et définir les traces internationales des chemins de Saint-Jacques.

Par analogie, s'agissant des itinéraires et réseaux ferroviaires, je pense que la même démarche sera possible, même s'il est vrai que chaque cas est spécifique et qu'il faudra justifier absolument l'intérêt national des protections proposées. On ne classera bien sûr pas tout le réseau même s'il est d'un intérêt évident, tant au niveau local que national, 
d'engager un véritable travail d'inventaire comme l'ont fait nos collègues suisses. Il permettra d'analyser la représentativité, la cohérence des protections de chaque réseau, ce que nous ne pouvons faire actuellement. Un réel travail de fond reste à engager pour déterminer la cohérence de ce qui est protégé au titre des lois de 1913 et de 1930 en fonction de la représentativité des types d'ouvrages et des modes d'impacts de ces équipements sur nos territoires. Pour cela, je souhaite faire appel à toutes les bonnes volontés pour nous aider dans cette démarche, pour nous signaler tout manquement grave de protections d'éléments du patrimoine existant. Je suis en effet tout à fait disposée à faire reconnaitre le caractère exceptionnel ou exemplaire de tel ou tel lieu ou objet technique et cela, en liaison avec nos collègues du ministère chargé de la Culture, pour utiliser les outils de protections les plus adaptés.

Je précise que ces processus de protection ne devraient pas se faire dans l'urgence. Ils nécessitent une appropriation locale mais également des expertises multiples, des enquêtes publiques qui demandent généralement de trois à cinq ans. Je considère que ce délai est légitime puisqu'il confère, à ce processus de reconnaissance et de validation des valeurs exceptionnelles, un caractère solennel très bien adapté au caractère définitif de cette protection. En effet, in fine, au Conseil d'État, nous tenons des discussions très approfondies sur le sens à donner à la protection, sur sa pérennité et sur sa justification scientifique.

L'intervention qui va suivre donnera quelques exemples des outils et relais dont nous disposons pour mener nos politiques de sites et paysages, à travers, notamment, l'action de nos directions régionales de l'environnement. En effet, ce sont nos principaux services déconcentrés qui, avec l'appui des services départementaux de l'architecture et du patrimoine, travaillent pour notre compte. Sur ce point, il existe souvent des confusions au niveau local, car ce sont des services locaux du ministère chargé de la Culture - les services départementaux de l'architecture et du patrimoine, en participant à la gestion au quotidien des protections des sites - qui interviennent parfois pour le ministère chargé de l'Écologie. 